Research Article

\title{
Singularity of Electromechanical Coupling Field in Piezoelectric Composite Junctions
}

\author{
Li Jiang $\mathbb{D}^{\mathbb{D}}$, Renyu Ge $\mathbb{D}^{\mathrm{D}}$, and Jinlun Zhang \\ School of Architecture and Civil Engineering, Anhui Polytechnic University, Wuhu, Anhui 241000, China \\ Correspondence should be addressed to Renyu Ge; geteam100@163.com
}

Received 21 December 2019; Accepted 3 February 2020; Published 25 February 2020

Academic Editor: Fabrizio Greco

Copyright (c) $2020 \mathrm{Li}$ Jiang et al. This is an open access article distributed under the Creative Commons Attribution License, which permits unrestricted use, distribution, and reproduction in any medium, provided the original work is properly cited.

\begin{abstract}
The double singularities including singular stress field and singular electric displacement field, in the tips of piezoelectric composite junctions, are analyzed by the interpolation matrix method (IMM). The double singularity analysis problem of piezoelectric composite junctions is converted into eigenvalue solution problem of ordinary differential equations with variable coefficients under corresponding boundary conditions. In numerical examples, the first couple of singularity orders and the corresponding characteristic angular functions of displacement and electric potential for the electromechanical coupling field are obtained and comparisons are presented to validate the accuracy of the proposed method. The singularity of the electromechanical coupling field at the tip of piezoelectric composite material junctions is closely related to the bonding angle and fiber direction. According to the numerical results, the best scheme can be configured for the combination of dissimilar materials.
\end{abstract}

\section{Introduction}

Piezoelectric devices such as brakes and sensors are composed of piezoelectric materials, conductors, and composite materials. The problem of material and geometric discontinuities is common at the junctions and interface edge of piezoelectric materials, conductors, and composite materials, which leads to the singularity of stress and electric displacement. The singular field at the tips of piezoelectric composite junctions directly affects the safety and engineering structures $[1,2]$. Therefore, it is of great significance to study the singularity of the notch to ensure the reliability and safety of the structure in service.

Since Williams [3] first gave the classical solution of stress field of interface crack in isotropic bimaterials, many research studies have been performed on the singularity in piezoelectric composite material wedges. The failure behavior and failure criteria of deep notch of piezoelectric ceramics were experimentally analyzed by Zhang et al. [4]. Liu and Chue [5] found that for some special notch angles of composite materials, the degree of stress singularity can be reduced or even disappeared by selecting the appropriate fiber direction. However, the experimental method is limited to obtaining at most the first two singular orders. In order to better understand the notch singularity, some numerical methods are applied to the research of notch singularity. Delale and Erdogan [6] and Chen and Harada [7] studied the stress singularity at the tip of cracks perpendicular to the interface of combined anisotropic materials and proposed the singular characteristic equations. Zhou et al. [8,9] adopted the Hamiltonian transformation method to analytically determine the stress and electrical strength factors of edge cracks for type III piezoelectric materials and investigate the Mode-III interface V-shape notch in a finite-size onedimensional hexagonal quasicrystalline bimaterial with piezoelectric effect in succession. In addition, Zhou et al. $[10,11]$ presented a symplectic approach based on the Hamiltonian system to analyze the electroelastic singularities and intensity factors of an interface crack in piezoelectric-elastic bimaterials and also to find the complex 
stress intensity factors and T-stress at an edge bimaterial interface crack.

Wippler and Kuna [12] used the boundary element method to analyze the singular electroelastic field at the tip of cracks of piezoelectric materials and pointed out that the problem of notch singularity is more complicated than that of crack. Stro transform was applied by Shen et al. [13] for the study of notch singularity of three-phase piezoelectric materials. Xu and Rajapakse [14] used the complex potential function method to investigate the singular electroelastic field at the tip of junctions of conductors, composite materials, and piezoelectric materials under plane deformation, and the influencing parameters including material properties, binding angle, and polarization angle were considered. But there are some defects in the analysis of interface crack by the complex potential function method. Chen et al. [15] made use of a modified one-dimensional finite element form to calculate the singular field of piezoelectric composite material junctions and that of the piezoelectric material and conductor junction. Li et al. [16] presented a semianalytical technique based on the scaled boundary finite element method to analyze two-dimensional cracks and notches inside piezoelectric composites. Luangarpa and Koguchi [17] extended a conservative integral based on the Betti reciprocal principle for calculating intensities of singularity at tips of interfaces in three-dimensional piezoelectric bonded joints. Islam et al. [18] evaluated the singularity orders in three-dimensional two-phase transversely isotropic piezoelectric dissimilar joints by the eigenanalysis method. Hrstka et al. [19] used the expanded Lekhnitskii-Eshelby-Stroh formalism to investigate an asymptotic in-plane problem of bimaterial sharp notches with various geometries and interface cracks in several generally monoclinic piezoelectric bimaterials.

In view of the above methods, transcendental equations are generally needed to be solved, and some of them cannot be degenerated to crack problems. Therefore, a new numerical method which adopts the interpolation matrix method is proposed to analyze the singularity at the tip of the notch. The displacement field at the tip of piezoelectric composite material junctions was firstly asymptotically expanded by power series, so that the electromechanical boundary/interface conditions of junctions were transformed into a combination expression of singularity-order characteristic equation. Based on the stress equilibrium equation and Maxwell equation, the characteristic equation about the singularity order of piezoelectric composite material junctions was derived. The developed IMM program [20-23] was used to solve the formed characteristic ordinary differential equations, which can calculate each singularity order and the corresponding characteristic angular function of displacement and electric potential for piezoelectric composite material junctions at one time. The present method has the characteristic of strong adaptability, high accuracy, and small calculation.

\section{Singularity in Wedge Notch of Piezoelectric Materials}

For the planar wedge notch of piezoelectric materials as shown in Figure 1, two rectangular coordinate systems (oxz and $\left.o x^{\prime} z^{\prime}\right)$ and one polar coordinate system (or $\left.\theta\right)$ are defined and the angle between the $z^{\prime}$ axis and $z$ axis is set as $\beta$.

In the rectangular coordinate system oxz, the constitutive relations for piezoelectric material domain (PZT) are expressed as follows:

$$
\left(\begin{array}{c}
\sigma_{x x} \\
\sigma_{z z} \\
\sigma_{x z} \\
D_{x} \\
D_{z}
\end{array}\right)=\left[\begin{array}{ccccc}
\widehat{c}_{11} & \widehat{c}_{13} & 0 & 0 & -\widehat{e}_{31} \\
\widehat{c}_{13} & \widehat{c}_{33} & 0 & 0 & -\widehat{e}_{33} \\
0 & 0 & \widehat{c}_{44} & -\widehat{e}_{15} & 0 \\
0 & 0 & \widehat{e}_{15} & \widehat{k}_{11} & 0 \\
\widehat{e}_{31} & \widehat{e}_{33} & 0 & 0 & \widehat{k}_{33}
\end{array}\right]\left(\begin{array}{c}
\varepsilon_{x x} \\
\varepsilon_{z z} \\
\varepsilon_{x z} \\
E_{x} \\
E_{z}
\end{array}\right),
$$

where $\widehat{c}_{11}, \widehat{c}_{13}, \widehat{c}_{33}$, and $\widehat{c}_{44}$ are the elastic modulus, $\widehat{e}_{15}, \widehat{e}_{31}$, and $\widehat{e}_{33}$ are the piezoelectric constant, and $\widehat{k}_{11}$ and $\widehat{k}_{33}$ are the dielectric constant. $\varepsilon_{i j}$ and $\sigma_{i j}(i j=x x, z z, x z)$ are the strain and stress, respectively. $E_{i}$ and $D_{i}$ are the electric field and electric displacement, respectively.

In the polar coordinate system or $\theta$, the constitutive relations can be expressed as

$$
\left\{\begin{array}{c}
\sigma_{r r} \\
\sigma_{\theta \theta} \\
\sigma_{r \theta} \\
D_{r} \\
D_{\theta}
\end{array}\right\}=\left[\begin{array}{lllll}
Q_{11} & Q_{12} & Q_{13} & Q_{14} & Q_{15} \\
Q_{21} & Q_{22} & Q_{23} & Q_{24} & Q_{25} \\
Q_{31} & Q_{32} & Q_{33} & Q_{34} & Q_{35} \\
Q_{41} & Q_{42} & Q_{43} & Q_{44} & Q_{45} \\
Q_{51} & Q_{52} & Q_{53} & Q_{54} & Q_{55}
\end{array}\right]\left\{\begin{array}{c}
\varepsilon_{r r} \\
\varepsilon_{\theta \theta} \\
\varepsilon_{r \theta} \\
E_{r} \\
E_{\theta}
\end{array}\right\},
$$

where $\varepsilon_{i j}$ and $\sigma_{i j}(i j=x x, z z, x z)$ are the strain and stress, respectively. $E_{i}$ and $D_{i}$ are the electric field and electric displacement, respectively. The matrix elements $Q_{i j}(i, j=$ $1, \ldots, 5)$ are the product of the elastic modulus $\left(\widehat{c}_{11}, \widehat{c}_{13}, \widehat{c}_{33}\right.$, and $\left.\widehat{c}_{44}\right)$, the piezoelectric constant $\left(\widehat{e}_{15}, \widehat{e}_{31}\right.$, and $\left.\widehat{e}_{33}\right)$ and the dielectric constant $\left(\widehat{k}_{11}\right.$ and $\left.\widehat{k}_{33}\right)$, and the $(\theta-\beta)$ trigonometric function [12], in which

$$
\left\{\begin{array}{c}
\varepsilon_{r r} \\
\varepsilon_{\theta \theta} \\
\varepsilon_{r \theta} \\
E_{r} \\
E_{\theta}
\end{array}\right\}=\left\{\begin{array}{c}
\frac{\partial u_{r}(r, \theta)}{\partial r} \\
\frac{u_{r}(r, \theta)}{r+\left(\partial u_{\theta}(r, \theta) /(r \partial \theta)\right)} \\
\frac{\partial u_{r}(r, \theta)}{(r \partial \theta)+\left(\partial u_{\theta}(r, \theta) /(\partial r)-\left(u_{\theta}(r, \theta) / r\right)\right)} \\
\frac{-\partial \varphi(r, \theta)}{\partial r} \\
\frac{-\partial \varphi(r, \theta)}{(r \partial \theta)}
\end{array}\right\} .
$$




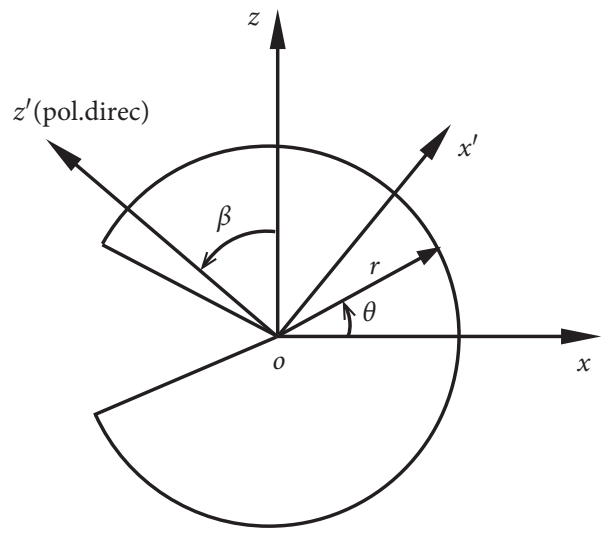

FIgURE 1: A piezoelectric wedge notch.

The displacement field $u_{r}(r, \theta)$ and potential field $\varphi(r, \theta)$ near the tip of the notch are expressed in the form of asymptotic expansion as follows [14]:

$$
\left\{\begin{array}{l}
u_{r}(r, \theta)=\sum_{k=1}^{n} A_{k} r^{\lambda_{k}} \widetilde{u}_{r k}(\theta), \\
u_{\theta}(r, \theta)=\sum_{k=1}^{n} A_{k} r^{\lambda_{k}} \widetilde{\mathcal{u}}_{\theta k}(\theta), \\
\varphi(r, \theta)=\sum_{k=1}^{n} A_{k} r^{\lambda_{k}} \widetilde{\varphi}_{k}(\theta),
\end{array}\right.
$$

where $r$ is the radial distance from the tip of notch, $A_{k}$ is the combined amplitude coefficient, $\lambda_{k}$ is the singularity order for the tip of notch, $n$ is the number of series terms intercepted, and $\widetilde{u}_{i k}(\theta)(i=r, \theta)$ and $\widetilde{\varphi}_{k}(\theta)$ are the characteristic angular function of displacement and characteristic angular function of electric potential, respectively.

In the absence of body force and free charge density, the equilibrium equations of the piezoelectric material are given by

$$
\left\{\begin{array}{c}
\nabla\left(r \widetilde{\sigma}_{r}\right)-\sigma_{\theta \theta} \\
\nabla\left(r \widetilde{\sigma}_{\theta}\right)+\sigma_{r \theta} \\
\nabla(r \mathbf{D})
\end{array}\right\}=\mathbf{0},
$$

where $\nabla=\{(\partial / \partial r),((1 / r)(\partial / \partial \theta))\}, \boldsymbol{\sigma}_{r}=\left\{\begin{array}{l}\sigma_{r r} \\ \sigma_{r \theta}\end{array}\right\}, \boldsymbol{\sigma}_{\theta}=\left\{\begin{array}{l}\sigma_{r \theta} \\ \sigma_{\theta \theta}\end{array}\right\}$, and $\mathbf{D}=\left\{\begin{array}{c}D_{r} \\ D_{\theta}\end{array}\right\}$

The typical terms $A_{k} r^{\lambda_{k}} \widetilde{\mathcal{u}}_{r k}(\theta), \quad A_{k} r^{\lambda_{k}} \widetilde{\mathcal{u}}_{\theta k}(\theta)$, and $A_{k} r^{r_{k}} \widetilde{\varphi}_{k}(\theta)$ in equation (4) are substituted into equations (2) and (3) and then substituted into equilibrium equation (5) of the piezoelectric material (for brevity, $\widetilde{u}_{i k}(\theta)(i=r, \theta)$, $\widetilde{\varphi}_{k}(\theta)$, and $\lambda_{k}$ are abbreviated as $\widetilde{u}_{i}(i=r, \theta), \widetilde{\varphi}$, and $\lambda$, respectively), and the equations can be obtained as follows:

$$
\begin{aligned}
& \widetilde{A}\left(\begin{array}{c}
\widetilde{u}_{r}^{\prime \prime}(\theta) \\
\widetilde{u}_{\theta}^{\prime \prime}(\theta) \\
\widetilde{\varphi}^{\prime \prime}(\theta)
\end{array}\right)+\widetilde{B}\left(\begin{array}{c}
\widetilde{u}_{r}^{\prime}(\theta) \\
\tilde{u}_{\theta}^{\prime}(\theta) \\
\widetilde{\varphi}^{\prime}(\theta)
\end{array}\right)+\widetilde{C}\left(\begin{array}{c}
\widetilde{u}_{r}(\theta) \\
\tilde{u}_{\theta}(\theta) \\
\widetilde{\varphi}(\theta)
\end{array}\right) \\
& +\lambda \widetilde{E}\left(\begin{array}{c}
\widetilde{u}_{r}^{\prime}(\theta) \\
\widetilde{u}_{\theta}^{\prime}(\theta) \\
\widetilde{\varphi}^{\prime}(\theta)
\end{array}\right)+\lambda \widetilde{F}\left(\begin{array}{c}
\widetilde{u}_{r}(\theta) \\
\tilde{u}_{\theta}(\theta) \\
\widetilde{\varphi}(\theta)
\end{array}\right) \\
& +\lambda^{2} \widetilde{G}\left(\begin{array}{c}
\widetilde{u}_{r}(\theta) \\
\tilde{u}_{\theta}(\theta) \\
\widetilde{\varphi}(\theta)
\end{array}\right)=\left\{\begin{array}{c}
0 \\
0 \\
0
\end{array}\right\},
\end{aligned}
$$

where

$$
\begin{aligned}
& \widetilde{A}=\left[\begin{array}{lll}
\widetilde{A}_{11}(\theta) & \widetilde{A}_{12}(\theta) & \widetilde{A}_{13}(\theta) \\
\widetilde{A}_{21}(\theta) & \widetilde{A}_{22}(\theta) & \widetilde{A}_{23}(\theta) \\
\widetilde{A}_{31}(\theta) & \widetilde{A}_{32}(\theta) & \widetilde{A}_{33}(\theta)
\end{array}\right] \quad \widetilde{B}=\left[\begin{array}{ccc}
\widetilde{B}_{11}(\theta) & \widetilde{B}_{12}(\theta) & \widetilde{B}_{13}(\theta) \\
\widetilde{B}_{21}(\theta) & \widetilde{B}_{22}(\theta) & \widetilde{B}_{23}(\theta) \\
\widetilde{B}_{31}(\theta) & \widetilde{B}_{32}(\theta) & \widetilde{B}_{33}(\theta)
\end{array}\right] \widetilde{C}=\left[\begin{array}{ccc}
\widetilde{C}_{11}(\theta) & \widetilde{C}_{12}(\theta) & \widetilde{C}_{13}(\theta) \\
\widetilde{C}_{21}(\theta) & \widetilde{C}_{22}(\theta) & \widetilde{C}_{23}(\theta) \\
\widetilde{C}_{31}(\theta) & \widetilde{C}_{32}(\theta) & \widetilde{C}_{33}(\theta)
\end{array}\right], \\
& \widetilde{E}=\left[\begin{array}{lll}
\widetilde{E}_{11}(\theta) & \widetilde{E}_{12}(\theta) & \widetilde{E}_{13}(\theta) \\
\widetilde{E}_{21}(\theta) & \widetilde{E}_{22}(\theta) & \widetilde{E}_{23}(\theta) \\
\widetilde{E}_{31}(\theta) & \widetilde{E}_{32}(\theta) & \widetilde{E}_{33}(\theta)
\end{array}\right] \quad \widetilde{F}=\left[\begin{array}{ccc}
\widetilde{F}_{11}(\theta) & \widetilde{F}_{12}(\theta) & \widetilde{F}_{13}(\theta) \\
\widetilde{F}_{21}(\theta) & \widetilde{F}_{22}(\theta) & \widetilde{F}_{23}(\theta) \\
\widetilde{F}_{31}(\theta) & \widetilde{F}_{32}(\theta) & \widetilde{F}_{33}(\theta)
\end{array}\right] \quad \widetilde{G}=\left[\begin{array}{ccc}
\widetilde{G}_{11}(\theta) & \widetilde{G}_{12}(\theta) & \widetilde{G}_{13}(\theta) \\
\widetilde{G}_{21}(\theta) & \widetilde{G}_{22}(\theta) & \widetilde{G}_{23}(\theta) \\
\widetilde{G}_{31}(\theta) & \widetilde{G}_{32}(\theta) & \widetilde{G}_{33}(\theta)
\end{array}\right],
\end{aligned}
$$


where matrix elements $\widetilde{A}_{i j}(\theta), \widetilde{B}_{i j}(\theta), \widetilde{C}_{i j}(\theta), \widetilde{E}_{i j}(\theta), \widetilde{F}_{i j}(\theta)$, and $\widetilde{G}_{i j}(\theta)$ are the linear combinations of the matrix element $Q_{i j}$ and its first derivative $Q_{i j}^{\prime}$ with respect to the coordinate $\theta .(\cdots)^{\prime}$ is the first derivative with respect to the coordinate $\theta$, and $(\cdots)^{\prime \prime}$ is the second derivative with respect to the coordinate $\theta$. The following is the same.

\section{Singularity in Wedge Notch of Composite Materials}

For the planar wedge notch of orthotropic composite materials as shown in Figure 2, the material principal axes are identified as axes 1 and 3. $\gamma$ is the angle between the principal axis system $(1,3)$ and the global coordinate system $(x, y)$ of the orthotropic material, that is, the direction angle of the composite fiber. $\theta$ is the angle between the polar coordinate system $(r, \theta)$ and global coordinate system $(x, y)$, which is positive in the counterclockwise direction.

According to the basic theory of elastic mechanics, in the case of plane stress, the stress-strain relationships of orthotropic materials in the principal axis system $(1,3)$ are as follows:

$$
\left\{\begin{array}{l}
\sigma_{11} \\
\sigma_{33} \\
\sigma_{13}
\end{array}\right\}=\left[\begin{array}{ccc}
C_{11} & C_{13} & 0 \\
C_{13} & C_{33} & 0 \\
0 & 0 & C_{66}
\end{array}\right]\left\{\begin{array}{c}
\varepsilon_{11} \\
\varepsilon_{33} \\
\varepsilon_{13}
\end{array}\right\},
$$

where $C_{i j}(i, j=1,2, \ldots, 6)$ is the stiffness coefficient matrix of composite materials in the principal axis system $(1,3)$, in which

$$
\begin{aligned}
& C_{11}=\frac{E_{1}}{1-v_{13} v_{31}}, \\
& C_{13}=\frac{v_{13} E_{3}}{1-v_{13} v_{31}}=\frac{v_{31} E_{1}}{1-v_{13} v_{31}}, \\
& C_{33}=\frac{E_{3}}{1-v_{13} v_{31}}, \\
& C_{66}=G_{13} .
\end{aligned}
$$

According to the coordinate transformation, the constitutive equation of orthotropic materials in the polar coordinate system $\operatorname{or} \theta$ can be expressed as

$$
\left(\begin{array}{l}
\Pi_{r r} \\
\Pi_{\theta \theta} \\
\Pi_{r \theta}
\end{array}\right)=\left[\begin{array}{lll}
D_{11} & D_{12} & D_{16} \\
D_{12} & D_{22} & D_{26} \\
D_{16} & D_{26} & D_{66}
\end{array}\right]\left(\begin{array}{c}
\Sigma_{r r} \\
\Sigma_{\theta \theta} \\
\Sigma_{r \theta}
\end{array}\right),
$$

where the matrix element $D_{i j}(i, j=1, \ldots, 6)$ is the product of material elastic moduli $E_{1}$ and $E_{3}$, shear modulus $G_{13}$, Poisson's ratios $v_{13}$ and $v_{31}$, and $\theta-\gamma$ trigonometric functions in the principal axis system $(1,3)$.

The strain geometry equation of orthotropic materials in polar coordinate system $\operatorname{or} \theta$ can be expressed as

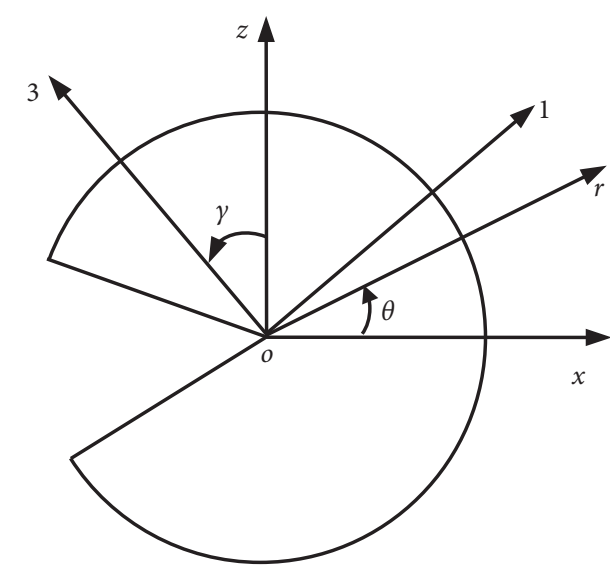

FIGURE 2: A wedge notch of orthotropic composite materials.

$$
\left(\begin{array}{c}
\Sigma_{r r} \\
\Sigma_{\theta \theta} \\
\Sigma_{r \theta}
\end{array}\right)=\left(\begin{array}{c}
\frac{\partial \psi_{r}(r, \theta)}{\partial r} \\
\frac{\psi_{r}(r, \theta)}{r}+\frac{1}{r} \frac{\partial \psi_{\theta}(r, \theta)}{\partial \theta} \\
\frac{1}{r} \frac{\partial \psi_{r}(r, \theta)}{\partial \theta}+\frac{\partial \psi_{\theta}(r, \theta)}{\partial r}-\frac{\psi_{\theta}(r, \theta)}{r}
\end{array}\right),
$$

where $\psi_{r}(r, \theta)$ and $\psi_{\theta}(r, \theta)$ are the displacement fields at the tip of wedge notch and $\Sigma_{r r}, \Sigma_{\theta \theta}$, and $\Sigma_{r \theta}$ are the stress field at the tip of the wedge notch.

The origin of the polar coordinate system is set at the tip of the notch and the displacement field of elastic deformation near the tip of the notch can be asymptotically expanded to a series of forms about radial direction [14]:

$$
\left\{\begin{array}{l}
\psi_{r}(r, \theta)=\sum_{k=1}^{n} A_{k} r^{\lambda_{k}} \widehat{\psi}_{r k}(\theta), \\
\psi_{\theta}(r, \theta)=\sum_{k=1}^{n} A_{k} r^{\lambda_{k}} \widehat{\psi}_{\theta k}(\theta),
\end{array}\right.
$$

where $\lambda_{k}$ is the stress singularity order, $\widehat{\psi}_{r k}(\theta)$ and $\widehat{\psi}_{\theta k}(\theta)$ are the characteristic angular function of displacement, $A_{k}$ is the corresponding displacement amplitude coefficient $(k=1$, $2, \ldots \ldots n)$, and $n$ is the number of series terms intercepted.

The equilibrium equations of the orthotropic composite materials without considering the body force are

$$
\left\{\begin{array}{l}
\nabla\left(r \Pi_{r}\right)-\Pi_{\theta \theta} \\
\nabla\left(r \Pi_{\theta}\right)+\Pi_{r \theta}
\end{array}\right\}=\mathbf{0}
$$

where $\quad \nabla=\{(\partial / \partial r),((1 / r)(\partial / \partial \theta))\}, \quad \Pi_{r}=\left\{\begin{array}{l}\Pi_{r r} \\ \Pi_{r \theta}\end{array}\right\}, \quad$ and $\Pi_{\theta}=\left\{\begin{array}{l}\Pi_{r \theta} \\ \Pi_{\theta \theta}\end{array}\right\}$.

The typical terms $A_{k} r^{\lambda_{k}} \widehat{\psi}_{r k}(\theta)$ and $A_{k} r^{\lambda_{k}} \widehat{\psi}_{\theta k}(\theta)$ in equation (12) are substituted into equations (10) and (11) and then substituted into the equilibrium equation (13). For brevity, $\widehat{\psi}_{i k}(\theta)(i=r, \theta)$ and $\lambda_{k}$ are abbreviated as $\widehat{\psi}_{i}(\theta)$ and $\lambda$, respectively, and the equations can be obtained as follows: 


$$
\begin{aligned}
& \hat{A}\left(\begin{array}{c}
\widehat{\psi}_{r}^{\prime \prime}(\theta) \\
\bar{\psi}_{\theta}^{\prime \prime}(\theta)
\end{array}\right)+\widehat{B}\left(\begin{array}{c}
\bar{\psi}_{r}^{\prime}(\theta) \\
\bar{\psi}_{\theta}^{\prime}(\theta)
\end{array}\right)+\bar{C}\left(\begin{array}{c}
\bar{\psi}_{r}(\theta) \\
\bar{\psi}_{\theta}(\theta)
\end{array}\right) \\
& +\lambda \widehat{E}\left(\begin{array}{c}
\bar{\psi}_{r}^{\prime}(\theta) \\
\bar{\psi}_{\theta}^{\prime}(\theta)
\end{array}\right)+\lambda \hat{F}\left(\begin{array}{c}
\bar{\psi}_{r}(\theta) \\
\bar{\psi}_{\theta}(\theta)
\end{array}\right) \\
& +\lambda^{2} \widehat{G}\left(\begin{array}{c}
\bar{\psi}_{r}(\theta) \\
\bar{\psi}_{\theta}(\theta)
\end{array}\right)=\left(\begin{array}{l}
0 \\
0
\end{array}\right),
\end{aligned}
$$

where

$$
\begin{aligned}
& \widehat{A}=\left[\begin{array}{ll}
\widehat{A}_{11}(\theta) & \widehat{A}_{12}(\theta) \\
\widehat{A}_{21}(\theta) & \widehat{A}_{22}(\theta)
\end{array}\right], \\
& \widehat{B}=\left[\begin{array}{ll}
\widehat{B}_{11}(\theta) & \widehat{B}_{12}(\theta) \\
\widehat{B}_{21}(\theta) & \widehat{B}_{22}(\theta)
\end{array}\right], \\
& \widehat{C}=\left[\begin{array}{ll}
\widehat{C}_{11}(\theta) & \widehat{C}_{12}(\theta) \\
\widehat{C}_{21}(\theta) & \widehat{C}_{22}(\theta)
\end{array}\right], \\
& \widehat{E}=\left[\begin{array}{ll}
\widehat{E}_{11}(\theta) & \widehat{E}_{12}(\theta) \\
\widehat{E}_{21}(\theta) & \widehat{E}_{22}(\theta)
\end{array}\right], \\
& \widehat{F}=\left[\begin{array}{ll}
\widehat{F}_{11}(\theta) & \widehat{F}_{12}(\theta) \\
\widehat{F}_{21}(\theta) & \widehat{F}_{22}(\theta)
\end{array}\right], \\
& \widehat{G}=\left[\begin{array}{ll}
\widehat{G}_{11}(\theta) & \widehat{G}_{12}(\theta) \\
\widehat{G}_{21}(\theta) & \widehat{G}_{22}(\theta)
\end{array}\right],
\end{aligned}
$$

where matrix elements $\widehat{A}_{i j}(\theta), \widehat{B}_{i j}(\theta), \widehat{C}_{i j}(\theta), \widehat{E}_{i j}(\theta), \widehat{F}_{i j}(\theta)$, and $\widehat{G}_{i j}(\theta)$ are the linear combinations of the matrix element $D_{i j}$ and its first derivative $D_{i j}^{\prime}$ with respect to the coordinate $\theta$.

\section{Singularity in Piezoelectric Composite Junctions}

As shown in Figure 3, the electromechanical boundary conditions for the two free boundaries of junctions are free stress and free electric displacement, i.e.,

When $\theta=\theta_{1}, \sigma_{\theta \theta}=\sigma_{r \theta}=0$, and $D_{\theta}=0$,

$$
\mathbf{R}_{1}\left(\begin{array}{c}
\widetilde{u}_{r}^{\prime}(\theta) \\
\widetilde{u}_{\theta}^{\prime}(\theta) \\
\widetilde{\varphi}^{\prime}(\theta)
\end{array}\right)+\mathbf{R}_{2}\left(\begin{array}{c}
\tilde{u}_{r}(\theta) \\
\widetilde{u}_{\theta}(\theta) \\
\widetilde{\varphi}(\theta)
\end{array}\right)+\lambda \mathbf{R}_{3}\left(\begin{array}{c}
\widetilde{u}_{r}(\theta) \\
\widetilde{u}_{\theta}(\theta) \\
\widetilde{\varphi}(\theta)
\end{array}\right)=\left(\begin{array}{l}
0 \\
0 \\
0
\end{array}\right),
$$

where $\mathbf{R}_{1}=\left[\begin{array}{lll}Q_{23} & Q_{22} & -Q_{25} \\ Q_{33} & Q_{32} & -Q_{35} \\ Q_{53} & Q_{52} & -Q_{55}\end{array}\right], \quad \mathbf{R}_{2}=\left[\begin{array}{lll}Q_{22} & -Q_{23} & 0 \\ Q_{32} & -Q_{33} & 0 \\ Q_{52} & -Q_{53} & 0\end{array}\right]$, and $\mathbf{R}_{3}=\left[\begin{array}{lll}Q_{21} & Q_{23} & -Q_{24} \\ Q_{31} & Q_{33} & -Q_{34} \\ Q_{51} & Q_{53} & -Q_{54}\end{array}\right]$.
When $\theta=\theta_{3}$, the stress is free, that is,

$$
\mathbf{S}_{1}\left(\begin{array}{c}
\hat{\psi}_{r}^{\prime}(\theta) \\
\bar{\psi}_{\theta}^{\prime}(\theta)
\end{array}\right)+\mathbf{S}_{2}\left(\begin{array}{c}
\hat{\psi}_{r}(\theta) \\
\hat{\psi}_{\theta}(\theta)
\end{array}\right)+\lambda \mathbf{S}_{3}\left(\begin{array}{c}
\hat{\psi}_{r}(\theta) \\
\hat{\psi}_{\theta}(\theta)
\end{array}\right)=\left(\begin{array}{l}
0 \\
0
\end{array}\right),
$$

where $\quad \mathbf{S}_{1}=\left[\begin{array}{ll}D_{26} & D_{22} \\ D_{66} & D_{26}\end{array}\right], \quad \mathbf{S}_{2}=\left[\begin{array}{ll}D_{22} & -D_{26} \\ D_{26} & -D_{66}\end{array}\right], \quad$ and $\mathbf{S}_{3}=\left[\begin{array}{ll}D_{12} & D_{26} \\ D_{16} & D_{66}\end{array}\right]$.

When $\theta=\theta_{2}$, the interface conditions between piezoelectric and composite materials are $\sigma_{\theta \theta}=\Pi_{\theta \theta}, \sigma_{r \theta}=\Pi_{r \theta}$, $D_{\theta}=0, u_{r}=\psi_{r}$, and $u_{\theta}=\psi_{\theta}$, that is,

$$
\mathbf{T}_{1}\left(\begin{array}{c}
\tilde{u}_{r}^{\prime}(\theta) \\
\tilde{u}_{\theta}^{\prime}(\theta) \\
\tilde{\varphi}^{\prime}(\theta) \\
\tilde{\psi}_{r}^{\prime}(\theta) \\
\bar{\psi}_{\theta}^{\prime}(\theta)
\end{array}\right)+\mathbf{T}_{2}\left(\begin{array}{c}
\tilde{u}_{r}(\theta) \\
\tilde{u}_{\theta}(\theta) \\
\widetilde{\varphi}(\theta) \\
\widehat{\psi}_{r}(\theta) \\
\bar{\psi}_{\theta}(\theta)
\end{array}\right)+\lambda \mathbf{T}_{3}\left(\begin{array}{c}
\tilde{u}_{r}(\theta) \\
\tilde{u}_{\theta}(\theta) \\
\widetilde{\varphi}(\theta) \\
\widehat{\psi}_{r}(\theta) \\
\bar{\psi}_{\theta}(\theta)
\end{array}\right)=\left(\begin{array}{l}
0 \\
0 \\
0 \\
0 \\
0
\end{array}\right),
$$

where

$$
\begin{aligned}
& \mathbf{T}_{1}=\left[\begin{array}{ccccc}
Q_{23} & Q_{22} & -Q_{25} & -D_{26} & -D_{22} \\
Q_{33} & Q_{32} & -Q_{35} & -D_{66} & -D_{26} \\
Q_{53} & Q_{52} & -Q_{55} & 0 & 0 \\
0 & 0 & 0 & 0 & 0 \\
0 & 0 & 0 & 0 & 0
\end{array}\right], \\
& \mathbf{T}_{2}=\left[\begin{array}{ccccc}
Q_{22} & -Q_{23} & 0 & -D_{22} & D_{26} \\
Q_{32} & -Q_{33} & 0 & -D_{26} & D_{66} \\
Q_{52} & -Q_{53} & 0 & 0 & 0 \\
1 & 0 & 0 & -1 & 0 \\
0 & 1 & 0 & 0 & -1
\end{array}\right], \\
& \mathbf{T}_{3}=\left[\begin{array}{ccccc}
Q_{21} & Q_{23} & -Q_{24} & -D_{12} & -D_{26} \\
Q_{31} & Q_{33} & -Q_{34} & -D_{16} & -D_{66} \\
Q_{51} & Q_{53} & -Q_{54} & 0 & 0 \\
0 & 0 & 0 & 0 & 0 \\
0 & 0 & 0 & 0 & 0
\end{array}\right] .
\end{aligned}
$$

Thus, the problem of calculating the singularity orders of the junctions bonded by piezoelectric and composite wedges is transformed into the problem of solving the eigenvalues of ordinary differential equations (6) and (14) under boundary conditions equations (16) and (17) and interface conditions equation (18). 


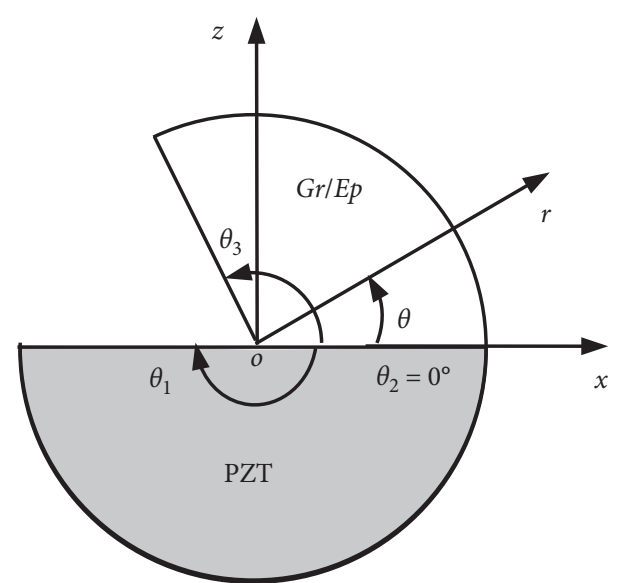

Figure 3: Configuration of piezoelectric-composite wedge junctions.

\section{Numerical Method}

As shown in Figure 3, the dimensionless coordinate $\xi$ is introduced into the wedge domain of piezoelectric material $\theta_{1} \leq \theta \leq \theta_{2}$ :

$$
\xi=\frac{\theta-\theta_{1}}{\theta_{2}-\theta_{1}}, \quad 0 \leq \xi \leq 1 .
$$

The characteristic angular functions of displacement, characteristic angular functions of electric potential, matrix elements $Q_{i j}$, and their derivatives of piezoelectric materials can be expressed as

$$
\begin{aligned}
& \left(\begin{array}{c}
\tilde{u}_{r}^{\prime \prime}(\theta) \\
\widetilde{u}_{\theta}^{\prime \prime}(\theta) \\
\widetilde{\varphi}^{\prime \prime}(\theta)
\end{array}\right)=\frac{1}{\left(\theta_{2}-\theta_{1}\right)^{2}}\left(\begin{array}{c}
\tilde{u}_{r}^{\prime \prime}(\xi) \\
\tilde{u}_{\theta}^{\prime \prime}(\xi) \\
\widetilde{\varphi}^{\prime \prime}(\xi)
\end{array}\right), \\
& \left(\begin{array}{c}
\widetilde{u}_{r}^{\prime}(\theta) \\
\widetilde{u}_{\theta}^{\prime}(\theta) \\
\widetilde{\varphi}^{\prime}(\theta)
\end{array}\right)=\frac{1}{\theta_{2}-\theta_{1}}\left(\begin{array}{c}
\widetilde{u}_{r}^{\prime}(\xi) \\
\widetilde{u}_{\theta}^{\prime}(\xi) \\
\widetilde{\varphi}^{\prime}(\xi)
\end{array}\right), \\
& \left(\begin{array}{c}
\widetilde{u}_{r}(\theta) \\
\widetilde{u}_{\theta}(\theta) \\
\widetilde{\varphi}(\theta)
\end{array}\right)=\left(\begin{array}{c}
\widetilde{u}_{r}(\xi) \\
\widetilde{u}_{\theta}(\xi) \\
\widetilde{\varphi}(\xi)
\end{array}\right), \\
& Q_{i j}^{\prime}(\theta)=\frac{1}{\theta_{2}-\theta_{1}} Q_{i j}^{\prime}(\xi), \\
& Q_{i j}(\theta)=Q_{i j}(\xi) \text {. }
\end{aligned}
$$

By substituting equations (21) and (22) into equation (6) for dimensionless coordinate, we can obtain

$$
\begin{gathered}
\frac{1}{\left(\theta_{2}-\theta_{1}\right)^{2}} \widetilde{A}\left(\begin{array}{c}
\tilde{u}_{r}^{\prime \prime}(\xi) \\
\widetilde{u}_{\theta}^{\prime \prime}(\xi) \\
\widetilde{\varphi}^{\prime \prime}(\xi)
\end{array}\right)+\frac{1}{\left(\theta_{2}-\theta_{1}\right)} \widetilde{B}\left(\begin{array}{c}
\tilde{u}_{r}^{\prime}(\xi) \\
\tilde{u}_{\theta}^{\prime}(\xi) \\
\widetilde{\varphi}^{\prime}(\xi)
\end{array}\right) \\
+\widetilde{C}\left(\begin{array}{c}
\widetilde{u}_{r}(\xi) \\
\tilde{u}_{\theta}(\xi) \\
\widetilde{\varphi}(\xi)
\end{array}\right)+\lambda \frac{1}{\left(\theta_{2}-\theta_{1}\right)} \widetilde{E}\left(\begin{array}{c}
\tilde{u}_{r}^{\prime}(\xi) \\
\widetilde{u}_{\theta}^{\prime}(\xi) \\
\widetilde{\varphi}^{\prime}(\xi)
\end{array}\right) \\
+\lambda \widetilde{F}\left(\begin{array}{c}
\widetilde{u}_{r}(\xi) \\
\widetilde{u}_{\theta}(\theta) \\
\widetilde{\varphi}(\theta)
\end{array}\right)+\lambda^{2} \widetilde{G}\left(\begin{array}{c}
\tilde{u}_{r}(\theta) \\
\widetilde{u}_{\theta}(\theta) \\
\widetilde{\varphi}(\theta)
\end{array}\right)=\left\{\begin{array}{l}
0 \\
0 \\
0
\end{array}\right\} .
\end{gathered}
$$

Then, the analysis of the electroelastic singularity field at the apex of the wedge of piezoelectric materials is transformed into the eigenvalue problem of ordinary differential equations with dimensionless coordinate $\xi$.

The dimensionless coordinate $\xi$ is introduced into the wedge domain of orthotropic composite material $\theta_{2} \leq \theta \leq \theta_{3}$ :

$$
\xi=\frac{\theta-\theta_{2}}{\theta_{3}-\theta_{2}},(0 \leq \xi \leq 1) .
$$

The characteristic angular functions of displacement, matrix elements $D_{i j}$, and their derivatives of composite materials can be expressed as

$$
\begin{aligned}
\left(\begin{array}{c}
\widehat{\psi}_{r}^{\prime \prime}(\theta) \\
\widehat{\psi}_{\theta}^{\prime \prime}(\theta)
\end{array}\right) & =\frac{1}{\left(\theta_{3}-\theta_{2}\right)^{2}}\left(\begin{array}{c}
\widehat{\psi}_{r}^{\prime \prime}(\xi) \\
\widehat{\psi}_{\theta}^{\prime \prime}(\xi)
\end{array}\right), \\
\left(\begin{array}{c}
\widehat{\psi}_{r}^{\prime}(\theta) \\
\widehat{\psi}_{\theta}^{\prime}(\theta)
\end{array}\right) & =\frac{1}{\theta_{3}-\theta_{2}}\left(\begin{array}{c}
\widehat{\psi}_{r}^{\prime}(\xi) \\
\widehat{\psi}_{\theta}^{\prime}(\xi)
\end{array}\right), \\
\left(\begin{array}{c}
\widehat{\psi}_{r}(\theta) \\
\widehat{\psi}_{\theta}(\theta)
\end{array}\right) & =\left(\begin{array}{c}
\widehat{\psi}_{r}(\xi) \\
\widehat{\psi}_{\theta}(\xi)
\end{array}\right), \\
D_{i j}^{\prime}(\theta) & =\frac{1}{\theta_{3}-\theta_{2}} D_{i j}^{\prime}(\xi), \\
D_{i j}(\theta) & =D_{i j}(\xi) .
\end{aligned}
$$

By substituting equations (25) and (26) into equation (14) for dimensionless coordinate, we can obtain

$$
\begin{aligned}
& \frac{1}{\left(\theta_{3}-\theta_{2}\right)^{2}} \hat{A}\left(\begin{array}{c}
\hat{\psi}_{r}^{\prime \prime}(\xi) \\
\hat{\psi}_{\theta}^{\prime \prime}(\xi)
\end{array}\right)+\frac{1}{\left(\theta_{3}-\theta_{2}\right)} \hat{B}\left(\begin{array}{c}
\hat{\psi}_{r}^{\prime}(\xi) \\
\widehat{\psi}_{\theta}^{\prime}(\xi)
\end{array}\right) \\
& +\widehat{C}\left(\begin{array}{c}
\widehat{\psi}_{r}(\xi) \\
\widehat{\psi}_{\theta}(\xi)
\end{array}\right)+\lambda \frac{1}{\left(\theta_{3}-\theta_{2}\right)} \hat{E}\left(\begin{array}{c}
\bar{\psi}_{r}^{\prime}(\xi) \\
\widehat{\psi}_{\theta}^{\prime}(\xi)
\end{array}\right) \\
& +\lambda \widehat{F}\left(\begin{array}{c}
\hat{\psi}_{r}(\xi) \\
\hat{\psi}_{\theta}(\xi)
\end{array}\right)+\lambda^{2} \widehat{G}\left(\begin{array}{c}
\hat{\psi}_{r}(\xi) \\
\hat{\psi}_{\theta}(\xi)
\end{array}\right)=\left(\begin{array}{l}
0 \\
0
\end{array}\right) .
\end{aligned}
$$


Then, the analysis of the singular stress field in wedge notch of orthotropic composite materials is transformed into the eigenvalue problem of ordinary differential equations with dimensionless coordinate $\xi$.
By introducing dimensionless coordinate $\xi$, the boundary and interface conditions are dimensionless and expressed as

$$
\begin{aligned}
& \frac{1}{\theta_{2}-\theta_{1}} \mathbf{R}_{1}\left(\begin{array}{c}
\tilde{u}_{r}^{\prime}(\xi) \\
\widetilde{u}_{\theta}^{\prime}(\xi) \\
\widetilde{\varphi}^{\prime}(\xi)
\end{array}\right)+\mathbf{R}_{2}\left(\begin{array}{c}
\tilde{u}_{r}(\xi) \\
\widetilde{u}_{\theta}(\xi) \\
\widetilde{\varphi}(\xi)
\end{array}\right)+\lambda \mathbf{R}_{3}\left(\begin{array}{c}
\tilde{u}_{r}(\xi) \\
\tilde{u}_{\theta}(\xi) \\
\widetilde{\varphi}(\xi)
\end{array}\right)=\left(\begin{array}{l}
0 \\
0 \\
0
\end{array}\right), \\
& \frac{1}{\theta_{3}-\theta_{2}} \mathbf{S}_{1}\left(\begin{array}{c}
\hat{\psi}_{r}^{\prime}(\xi) \\
\bar{\psi}_{\theta}^{\prime}(\xi)
\end{array}\right)+\mathbf{S}_{2}\left(\begin{array}{c}
\widehat{\psi}_{r}(\xi) \\
\widehat{\psi}_{\theta}(\xi)
\end{array}\right)+\lambda \mathbf{S}_{3}\left(\begin{array}{c}
\widehat{\psi}_{r}(\xi) \\
\widehat{\psi}_{\theta}(\xi)
\end{array}\right)=\left(\begin{array}{l}
0 \\
0
\end{array}\right),
\end{aligned}
$$

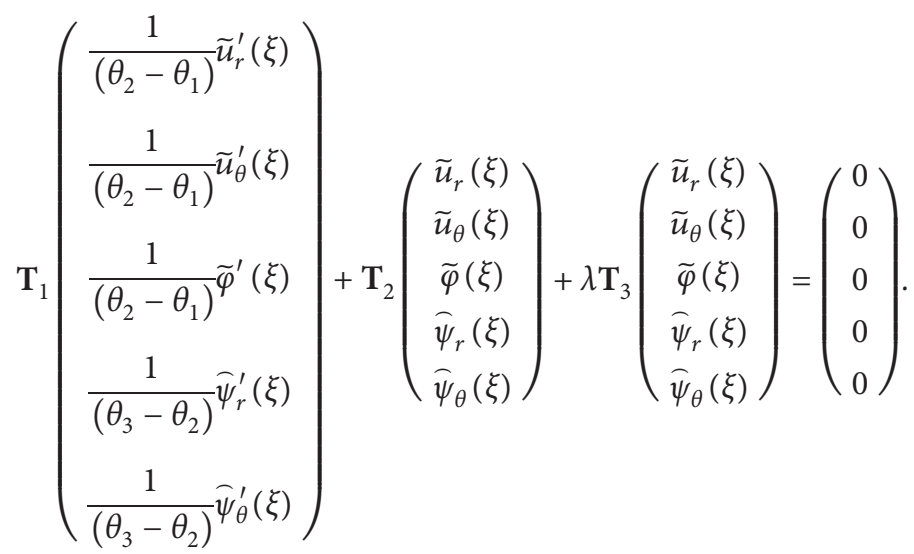

The wedge domains $\theta_{1} \leq \theta \leq \theta_{2}$ and $\theta_{2} \leq \theta \leq \theta_{3}$ are transformed into dimensionless region $0 \leq \xi \leq 1$, and the interval $[0,1]$ is divided into $N$ segments, $0=\xi_{0}, \xi_{1}$, $\xi_{2}, \ldots, \xi_{n-1}, \xi_{n}=1$, and $\Delta l_{i}=\xi_{i}-\xi_{i-1}=1 / N$. By using the difference method, the derivative values of the five functions $\widetilde{u}_{r}(\xi), \widetilde{u}_{\theta}(\xi), \widetilde{\varphi}(\xi), \widehat{\psi}_{r}(\xi)$, and $\widehat{\psi}_{\theta}(\xi)$ in the system of ordinary differential equations with variable coefficients are expressed by the function values on the interval partition points:

$$
\left\{\begin{array}{l}
\widetilde{u}_{r}^{\prime}\left(\xi_{j}\right)-\widetilde{u}_{r}^{\prime}\left(\xi_{0}\right)=\int_{\xi_{0}}^{\xi_{j}} \widetilde{u}_{r}^{\prime \prime}(\xi) \mathrm{d} \xi, \\
\widetilde{u}_{\theta}^{\prime}\left(\xi_{j}\right)-\widetilde{u}_{\theta}^{\prime}\left(\xi_{0}\right)=\int_{\xi_{0}}^{\xi_{j}} \widetilde{u}_{\theta}^{\prime \prime}(\xi) \mathrm{d} \xi, \\
\widetilde{\varphi}^{\prime}\left(\xi_{j}\right)-\widetilde{\varphi}^{\prime}\left(\xi_{0}\right)=\int_{\xi_{0}}^{\xi_{j}} \widetilde{\varphi}^{\prime \prime}(\xi) \mathrm{d} \xi, \quad(j=0,1,2, \ldots, n) . \\
\widehat{\psi}_{r}^{\prime}\left(\xi_{j}\right)-\widehat{\psi}_{r}^{\prime}\left(\xi_{0}\right)=\int_{\xi_{0}}^{\xi_{j}} \widetilde{\psi}_{r}^{\prime \prime}(\xi) \mathrm{d} \xi, \\
\widehat{\psi}_{\theta}^{\prime}\left(\xi_{j}\right)-\widehat{\psi}_{\theta}^{\prime}\left(\xi_{0}\right)=\int_{\xi_{0}}^{\xi_{j}} \widehat{\psi}_{\theta}^{\prime \prime}(\xi) \mathrm{d} \xi,
\end{array}\right.
$$

In the above formula, $\widetilde{u}_{r}^{\prime \prime}(\xi), \widetilde{u}_{\theta}^{\prime \prime}(\xi), \widetilde{\varphi}^{\prime \prime}(\xi), \widehat{\psi}_{r}^{\prime \prime}(\xi)$, and $\bar{\psi}_{\theta}^{\prime \prime}(\xi)$ are approximated by interpolation functions without loss of generality. Take function $\widetilde{u}_{r}^{\prime \prime}(\xi)$ as an example to illustrate 


$$
\widetilde{u}_{r}^{\prime \prime}(\xi)=\sum_{i=1}^{N} \widetilde{u}_{r}^{\prime \prime}\left(\xi_{i}\right) L_{i}(\xi)
$$

where $L_{i}(\xi)$ is the Lagrange interpolation basis function, so

$$
\begin{aligned}
\tilde{u}_{r}^{\prime}\left(\xi_{j}\right) & -\tilde{u}_{r}^{\prime}\left(\xi_{0}\right)=\sum_{i=0}^{n} \tilde{u}_{r}^{\prime \prime}\left(\xi_{j}\right) J_{j i}, \\
& i=0,1,2, \ldots, N ; j=0,1,2, \ldots, N,
\end{aligned}
$$

where $J_{j i}=\int_{\xi_{0}}^{\xi_{j}} L_{i}(\xi) \mathrm{d} \xi$, and vectors and matrix notations are introduced

$$
\begin{aligned}
\boldsymbol{\tau} & =\left\{\begin{array}{c}
0 \\
0 \\
\vdots \\
0
\end{array}\right\}, \\
\boldsymbol{\sigma} & =\left\{\begin{array}{c}
1 \\
1 \\
\vdots \\
1
\end{array}\right\}, \\
\mathbf{J} & =\left[\begin{array}{ccccc}
0 & 0 & 0 & \cdots & 0 \\
J_{10} & J_{11} & J_{12} & \cdots & J_{1 n} \\
J_{20} & J_{21} & J_{22} & \cdots & J_{2 n} \\
\cdots & \cdots & \cdots & \cdots & \cdots \\
J_{n 0} & J_{n 1} & J_{n 2} & \cdots & J_{n n}
\end{array}\right], \\
\mathbf{I} & =\left[\begin{array}{ccccc}
1 & 0 & 0 & \cdots & 0 \\
0 & 1 & 0 & \cdots & 0 \\
0 & 0 & 1 & \cdots & 0 \\
\cdots & \cdots & \cdots & \cdots & \cdots \\
0 & 0 & 0 & \cdots & 1
\end{array}\right],
\end{aligned}
$$

where the matrix $\mathbf{J}$ is called as the integral matrix, which only depends on the interpolation basis function $L_{i}(\xi)$. Quadratic parabola interpolation is adopted, and the basis function is expressed as

$$
L_{i}(\xi)=\left\{\begin{array}{l}
\frac{\left(\xi-\xi_{i}\right)\left(\xi-\xi_{i+1}\right)}{2\left(\Delta l_{i}\right)^{2}} \\
\frac{\left(\xi-\xi_{i-1}\right)\left(\xi-\xi_{i+1}\right)}{-\left(\Delta l_{i}\right)^{2}}, \quad \xi \in\left[\xi_{i-1}, \xi_{i+1}\right] . \\
\frac{\left(\xi-\xi_{i-1}\right)\left(\xi-\xi_{i}\right)}{2\left(\Delta l_{i}\right)^{2}}
\end{array}\right.
$$

The function $\tilde{u}_{r}(\xi)$ and its derivative functions of each order in equation (31) are written in the vector form as follows:

$$
\begin{aligned}
\widetilde{u}_{r}^{\prime}\left(\xi_{i}\right) & =\boldsymbol{\tau} \widetilde{u}_{r}\left(\xi_{0}\right)+\boldsymbol{\sigma} \widetilde{u}_{r}^{\prime}\left(\xi_{0}\right)+\mathbf{J} \widetilde{u}_{r}^{\prime \prime}\left(\xi_{i}\right) \\
& =[\boldsymbol{\tau}, \boldsymbol{\sigma}, \mathbf{J}]_{(N+1) \times(N+3)}\left(\begin{array}{c}
\widetilde{u}_{r}\left(\xi_{0}\right) \\
\tilde{u}_{r}^{\prime}\left(\xi_{0}\right) \\
\tilde{u}_{r}^{\prime \prime}\left(\xi_{i}\right)
\end{array}\right)_{(N+3) \times 1} .
\end{aligned}
$$

Let

$$
\begin{aligned}
\mathbf{H}_{1} & =[\boldsymbol{\tau}, \boldsymbol{\sigma}, \mathbf{J}]_{(n+1) \times(n+3)}, \\
\tilde{Y}_{r} & =\left(\begin{array}{c}
\widetilde{u}_{r}\left(\xi_{0}\right) \\
\tilde{u}_{r}^{\prime \prime}\left(\xi_{0}\right) \\
\tilde{u}_{r}^{\prime \prime}\left(\xi_{i}\right)
\end{array}\right)_{(N+3 \times 1)},
\end{aligned}
$$

such that equation (36) can be expressed as

$$
\tilde{u}_{r}^{\prime}\left(\xi_{i}\right)=\mathbf{H}_{1} \widetilde{Y}_{r}
$$

Then, the low-order derivative function is replaced by the higher-order derivative function in sequence, and the following formula can be obtained by stepwise recursion:

$$
\begin{aligned}
\tilde{u}_{r}\left(\xi_{i}\right) & =\boldsymbol{\sigma} \widetilde{u}_{r}\left(\xi_{0}\right)+\mathbf{J} \boldsymbol{\sigma} \widetilde{u}_{r}^{\prime}\left(\xi_{0}\right)+\mathbf{J}^{2} \widetilde{u}_{r}^{\prime \prime}\left(\xi_{i}\right) \\
& =\left[\boldsymbol{\sigma}, \mathbf{J} \boldsymbol{\sigma}, \mathbf{J}^{2}\right]_{(N+1) \times(N+3)}\left(\begin{array}{c}
\tilde{u}_{r}\left(\xi_{0}\right) \\
\tilde{u}_{r}^{\prime}\left(\xi_{0}\right) \\
\tilde{u}_{r}^{\prime \prime}\left(\xi_{i}\right)
\end{array}\right)_{(N+3) \times 1} .
\end{aligned}
$$

Let

$$
\mathbf{H}_{0}=\left[\boldsymbol{\sigma}, \mathbf{J} \boldsymbol{\sigma}, \mathbf{J}^{2}\right]_{(N+1) \times(N+3)} .
$$

such that equation (39) can be expressed as

$$
\begin{aligned}
\tilde{u}_{r}\left(\xi_{i}\right) & =\mathbf{H}_{0} \tilde{Y}_{r}, \\
\tilde{u}_{r}^{\prime \prime}\left(\xi_{i}\right) & =\boldsymbol{\tau} \widetilde{u}_{r}\left(\xi_{0}\right)+\boldsymbol{\tau} \widetilde{u}_{r}^{\prime}\left(\xi_{0}\right)+\mathbf{I} \widetilde{u}_{r}^{\prime \prime}\left(\xi_{i}\right) \\
& =[\boldsymbol{\tau}, \boldsymbol{\tau}, \mathbf{I}]_{(N+1) \times(N+3)}\left(\begin{array}{c}
\tilde{u}_{r}\left(\xi_{0}\right) \\
\tilde{u}_{r}^{\prime}\left(\xi_{0}\right) \\
\tilde{u}_{r}^{\prime \prime}\left(\xi_{i}\right)
\end{array}\right)_{(N+3) \times 1} .
\end{aligned}
$$

Equation (41) can be expressed as

$$
\begin{aligned}
\mathbf{H}_{2} & =[\boldsymbol{\tau}, \boldsymbol{\tau}, \mathbf{I}]_{(N+1) \times(N+3)}, \\
\tilde{u}_{r}^{\prime \prime}\left(\xi_{i}\right) & =\mathbf{H}_{2} \widetilde{Y}_{r} .
\end{aligned}
$$

The other function vectors are handled in the same way and given by 


$$
\begin{aligned}
\widetilde{u}_{\theta}\left(\xi_{i}\right) & =\mathbf{H}_{0} \widetilde{Y}_{\theta}, \\
\widetilde{u}_{\theta}^{\prime}\left(\xi_{i}\right) & =\mathbf{H}_{1} \widetilde{Y}_{\theta}, \\
\widetilde{u}_{\theta}^{\prime \prime}\left(\xi_{i}\right) & =\mathbf{H}_{2} \widetilde{Y}_{\theta}, \\
\widetilde{\varphi}\left(\xi_{i}\right) & =\mathbf{H}_{0} \widetilde{Y}_{\varphi}, \\
\widetilde{\varphi}^{\prime}\left(\xi_{i}\right) & =\mathbf{H}_{1} \widetilde{Y}_{\varphi}, \\
\widetilde{\varphi}^{\prime \prime}\left(\xi_{i}\right) & =\mathbf{H}_{2} \widetilde{Y}_{\varphi}, \\
\widehat{\psi}_{r}\left(\xi_{i}\right) & =\mathbf{H}_{0} \widehat{Y}_{r}, \\
\widehat{\psi}_{r}^{\prime}\left(\xi_{i}\right) & =\mathbf{H}_{1} \widehat{Y}_{r}, \\
\widehat{\psi}_{r}^{\prime \prime}\left(\xi_{i}\right) & =\mathbf{H}_{2} \widehat{Y}_{r}, \\
\widehat{\psi}_{\theta}\left(\xi_{i}\right) & =\mathbf{H}_{0} \widehat{Y}_{\theta}, \\
\widehat{\psi}_{\theta}^{\prime}\left(\xi_{i}\right) & =\mathbf{H}_{1} \widehat{Y}_{\theta}, \\
\widehat{\psi}_{\theta}^{\prime \prime}\left(\xi_{i}\right) & =\mathbf{H}_{2} \widehat{Y}_{\theta},
\end{aligned}
$$

where

$$
\begin{aligned}
& \widetilde{Y}_{\theta}=\left(\begin{array}{l}
\widetilde{u}_{\theta}\left(\xi_{0}\right) \\
\tilde{u}_{\theta}^{\prime}\left(\xi_{0}\right) \\
\tilde{u}_{\theta}^{\prime \prime}\left(\xi_{i}\right)
\end{array}\right)_{(N+3) \times 1}, \\
& \widetilde{Y}_{\varphi}=\left(\begin{array}{c}
\widetilde{\varphi}\left(\xi_{0}\right) \\
\widetilde{\varphi}^{\prime}\left(\xi_{0}\right) \\
\widetilde{\varphi}^{\prime \prime}\left(\xi_{i}\right)
\end{array}\right)_{(N+3) \times 1}, \\
& \hat{Y}_{r}=\left(\begin{array}{c}
\hat{\psi}_{r}\left(\xi_{0}\right) \\
\bar{\psi}_{r}^{\prime}\left(\xi_{0}\right) \\
\bar{\psi}_{r}^{\prime}\left(\xi_{i}\right)
\end{array}\right)_{(N+3) \times 1}, \\
& \hat{Y}_{\theta}=\left(\begin{array}{c}
\hat{\psi}_{\theta}\left(\xi_{0}\right) \\
\hat{\psi}_{\theta}^{\prime}\left(\xi_{0}\right) \\
\hat{\psi}_{\theta}^{\prime \prime}\left(\xi_{i}\right)
\end{array}\right)_{(N+3) \times 1} .
\end{aligned}
$$

Each coefficient in equations (23), (27), and (28)-(30) is written as a diagonal matrix of $(N+1) \times(N+1)$. For example, $\widetilde{A}_{i j}(\xi)$ and $\widehat{A}_{i j}(\xi)$ are transformed into the diagonal matrix after discretization, and others are similar:

$$
\begin{gathered}
\widetilde{A}_{i j}=\left[\begin{array}{ccccc}
\widetilde{A}_{i j}\left(\xi_{0}\right) & 0 & 0 & \ldots & 0 \\
0 & \widetilde{A}_{i j}\left(\xi_{1}\right) & 0 & \ldots & 0 \\
0 & 0 & \tilde{A}_{i j}\left(\xi_{3}\right) & \ldots & 0 \\
\ldots & \ldots & \ldots & \ldots & \ldots \\
0 & 0 & 0 & \ldots & \widetilde{A}_{i j}\left(\xi_{N}\right)
\end{array}\right], \\
\widehat{A}_{i j}=\left[\begin{array}{ccccc}
\widehat{A}_{i j}\left(\xi_{0}\right) & 0 & 0 & \ldots & 0 \\
0 & \widehat{A}_{i j}\left(\xi_{1}\right) & 0 & \ldots & 0 \\
0 & 0 & \widehat{A}_{i j}\left(\xi_{3}\right) & \ldots & 0 \\
\ldots & \ldots & \ldots & \ldots & \ldots \\
0 & 0 & 0 & \ldots & \widehat{A}_{i j}\left(\xi_{N}\right)
\end{array}\right] .
\end{gathered}
$$

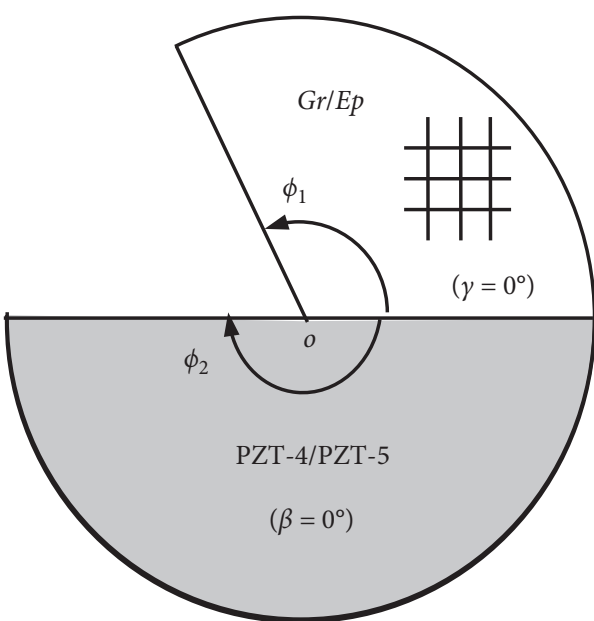

Figure 4: Geometry for piezoelectric-composite wedge junctions.

By substituting equations (43)-(45) into equations (23), (27), and (28)-(30), the calculation of the singularity order of the electromechanical coupling field at the tip of piezoelectric composite material junctions is transformed into a set of algebraic equation eigenvalue problems:

$$
\mathbf{L Y}+\lambda \mathbf{M Y}=\mathbf{0},
$$

where $\mathbf{Y}=\left(\begin{array}{lllll}\tilde{Y}_{r} & \widetilde{Y}_{\theta} & \tilde{Y}_{\varphi} & \widehat{Y}_{r} & \widehat{Y}_{\theta}\end{array}\right)^{T}$, the matrices $\mathbf{L}$ and $\mathbf{M}$ are the linear combinations of the diagonal matrices $\widetilde{A}_{i j}, \widetilde{B}_{i j}, \widetilde{C}_{i j}$, ... and $\widehat{A}_{i j}, \widehat{B}_{i j}, \widehat{C}_{i j}, \ldots$ Equation (46) is a general characteristic equation set of $5(n+3)$ unknown vectors $\mathbf{Y}=\left(\begin{array}{lllll}\tilde{Y}_{r} & \tilde{Y}_{\theta} & \tilde{Y}_{\varphi} & \bar{Y}_{r} & \bar{Y}_{\theta}\end{array}\right)^{T}$. By using the QR method to solve the equation, many eigenvalues $\lambda$ and corresponding eigenvectors $\mathbf{Y}=\left(\begin{array}{llllll}\tilde{Y}_{r} & \tilde{Y}_{\theta} & \tilde{Y}_{\varphi} & \bar{Y}_{r} & \bar{Y}_{\theta}\end{array}\right)^{T}$ can be obtained.

\section{Example Analysis and Discussion}

The piezoelectric composite material wedge junction is shown in Figure 4. The junction angle of PZT-4/PZT-5 piezoelectric material wedge notch is $\phi_{2}=180^{\circ}$. The junction angle of composite material wedge notch is within the range $0^{\circ}<\phi_{1} \leq 180^{\circ}$. When $\phi_{1}=180^{\circ}$, the bimaterial junction structure degenerates into interface crack structure. The first four-order values of the stress and electric displacement singular characteristic order in the interface crack tip region are shown in Table 1 by using the interpolation matrix method (IMM). In Table 1, $N$ refers to the number of segments at the tip of each wedge notch. The unknown function in the subinterval is simulated by quadratic piecewise polynomial function. The more segments $N$ is, the higher the calculation accuracy is, and $\operatorname{Re}\left(\lambda_{1}-1\right)$ converges to -0.500000 . Here, $-1<\operatorname{Re}\left(\lambda_{1}-1\right)<0$ is the singular term and $\operatorname{Re}\left(\lambda_{2}-1\right)>0, \operatorname{Re}\left(\lambda_{3}-1\right)>0$, and $\operatorname{Re}\left(\lambda_{4}-1\right)>0$ are the nonsingular terms. These nonsingular parameters are indispensable to describe the complete stress field at the tip of piezoelectric composite material junctions [24]. At present, there is still a lack of these singular order values for 
TAвLE 1: The first four singularity orders of the interface crack with interval segmentation number $N$.

\begin{tabular}{lcccc}
\hline$N$ & $\lambda_{1}-1$ & $\lambda_{2}-1$ & $\lambda_{3}-1$ & $\lambda_{4}-1$ \\
\hline 10 & $-0.510769-0.0406356 i$ & $-0.510769+0.0406356 i$ & $0.504289-0.0455896 i$ & $0.504289+0.0455896 i$ \\
20 & $-0.502625-0.0448070 i$ & $-0.502625+0.0448070 i$ & $0.500793-0.0440447 i$ & $0.500793+0.0440447 i$ \\
30 & $-0.500487-0.0444804 i$ & $-0.500487+0.044480 \mathrm{a} 4 i$ & $0.500149-0.0440330 i$ & $0.500149+0.0440330 i$ \\
40 & $-0.500063-0.0442202 i$ & $-0.500063+0.0442202 i$ & $0.500035-0.0440385 i$ & $0.500035+0.0440385 i$ \\
50 & $-0.499986-0.0441066 i$ & $-0.499986+0.0441066 i$ & $0.500010-0.0440370 i$ & $0.500010+0.0440370 i$ \\
60 & $-0.499978-0.0440604 i$ & $-0.499978+0.0440604 i$ & $0.500003-0.0440345 i$ & $0.500003+0.0440345 i$ \\
70 & $-0.499982-0.0440414 i$ & $-0.499982+0.0440414 i$ & $0.500001-0.0440325 i$ & $0.500001+0.0440325 i$ \\
80 & $-0.499987-0.0440334 i$ & $-0.499987+0.0440334 i$ & $0.500000-0.0440311 i$ & $0.500000+0.0440311 i$ \\
Ref. [15] & $-0.5-0.044 i$ & $-0.5+0.044 i$ & - & - \\
\hline
\end{tabular}

Note: $i=\sqrt{-1}$ is the imaginary number.

TABLE 2: The first four singularity orders of piezoelectric composite material wedge junction with interval segmentation number $N$, when $\phi_{1}=90^{\circ}$.

\begin{tabular}{lcccc}
\hline$N$ & $\lambda_{1}-1$ & $\lambda_{2}-1$ & $\lambda_{3}-1$ & $\lambda_{4}-1$ \\
\hline 10 & -0.34958611 & -0.022406747 & $0.62279008-0.38780841 i$ & $0.6227900+0.387808418 i$ \\
20 & -0.34989329 & -0.022582134 & $0.62231249-0.387750664 i$ & $0.62231249+0.387750664 i$ \\
40 & -0.34997618 & -0.022601267 & $0.62225081-0.38760694 i$ & $0.62225081+0.38760694 i$ \\
80 & -0.34998674 & -0.022600726 & $0.62224740-0.38759404 i$ & $0.62224740+0.38759404 i$ \\
Ref. [15] & -0.34998 & -0.0226004 & - & - \\
\hline
\end{tabular}

Note: $i=\sqrt{-1}$ is the imaginary number.

the tip of piezoelectric composite material junctions, especially for the region far from the tip of junctions. When $\phi_{1}=90^{\circ}$, the first four-order values of the stress singular characteristic order in the bimaterial junctions are shown in Table 2. The results obtained in this paper are in good agreement with the calculated results in the existing literature [15].

As shown in Figure 4, the junction angle is $\phi_{2}=180^{\circ}$ and the polarization angle of piezoelectric material PZT-4/ PZT-5 is $\beta=0^{\circ}$. When the junction angle of composite wedge notch is $0^{\circ}<\phi_{1} \leq 180^{\circ}$ and the number of segments $N=40$, the order values are calculated by the interpolation matrix method. Figure 5 shows the variations of singularity orders $\lambda_{k}-1$ with junction angle $\phi_{1}$ for the electromechanical coupling field of the junctions between piezoelectric material PZT-4/PZT-5 and composite material $\mathrm{Gr} / \mathrm{Ep}$. It can be seen that the calculated values are consistent with the solution in the literature [15], indicating the validity and accuracy of the calculated method in this paper. At the same time, Figure 5 also shows that with the increase of the junction angle $\phi_{1}$, the first two singularity orders $\lambda_{k}-1$ of the coupling field are negative and decrease gradually, that is, the singularity of the electromechanical coupling field becomes stronger. When $\phi_{1}=180^{\circ}$, the piezoelectric composite material junctions shown in Figure 4 degenerate into the interface crack model, the singularity of the electromechanical coupling field at the tip of junctions is the strongest, and the real part of the singularity order is $\operatorname{Re}\left(\lambda_{1}-1\right)=-0.5000638$.

The method in this paper can not only obtain the useful singularity orders of stress and electric displacement at the tip of piezoelectric and composite material junctions but can also obtain the corresponding characteristic angular functions of displacement and electric potential and their

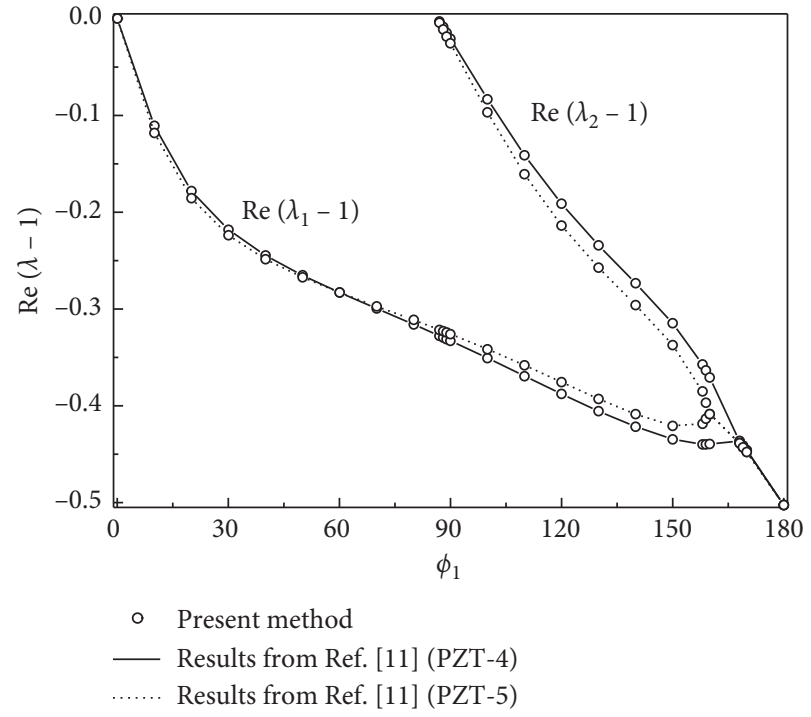

FIgURE 5: The variations of singularity orders with $\phi_{1}$ for piezoelectric-composite wedge junctions.

derivative functions. Figure 6 shows the curves of characteristic angular function corresponding to the first stress singularity order $\lambda_{1}-1=-0.2828998$ of $\mathrm{PZT}-4-\mathrm{Gr} / \mathrm{Ep}$ junction at $\phi_{1}=60^{\circ}, \phi_{2}=180^{\circ}$, and $N=40$. Figure 7 shows the curves of the first derivative function of the characteristic angular function corresponding to the first stress singularity order $\lambda_{1}-1=-0.2828998$ of $\mathrm{PZT}-4-\mathrm{Gr} / \mathrm{Ep}$ junction at $\phi_{1}=60^{\circ}, \phi_{2}=180^{\circ}$, and $N=40$. It can be seen from Figures 6 and 7 that the characteristic angular functions of displacement and electric potential are continuous at the bonding position of dissimilar materials, and their first derivative functions change suddenly. These 


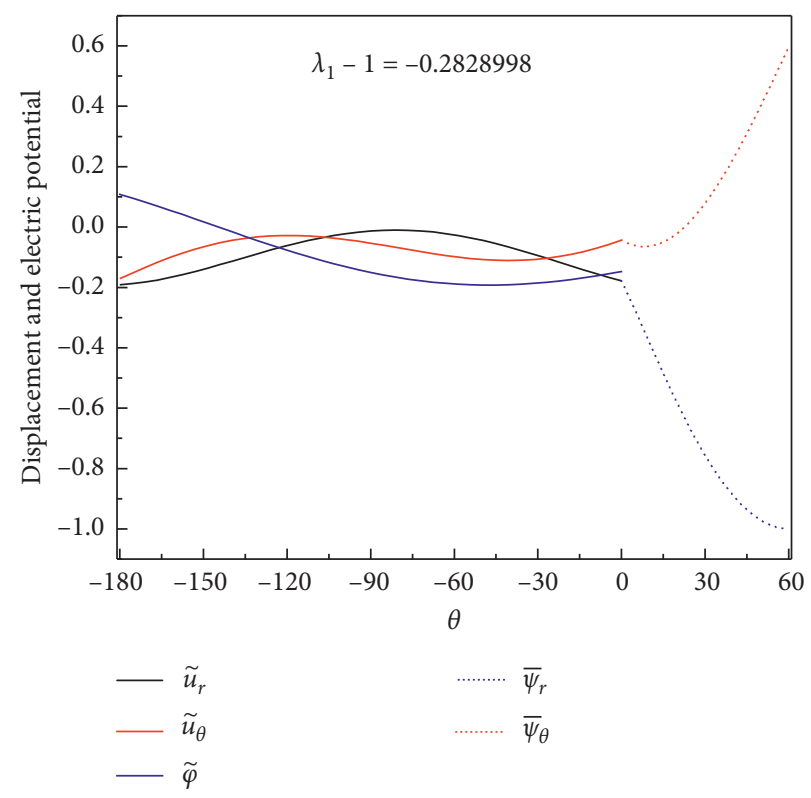

FIgURE 6: The characteristic angular function of displacement and electric potential.

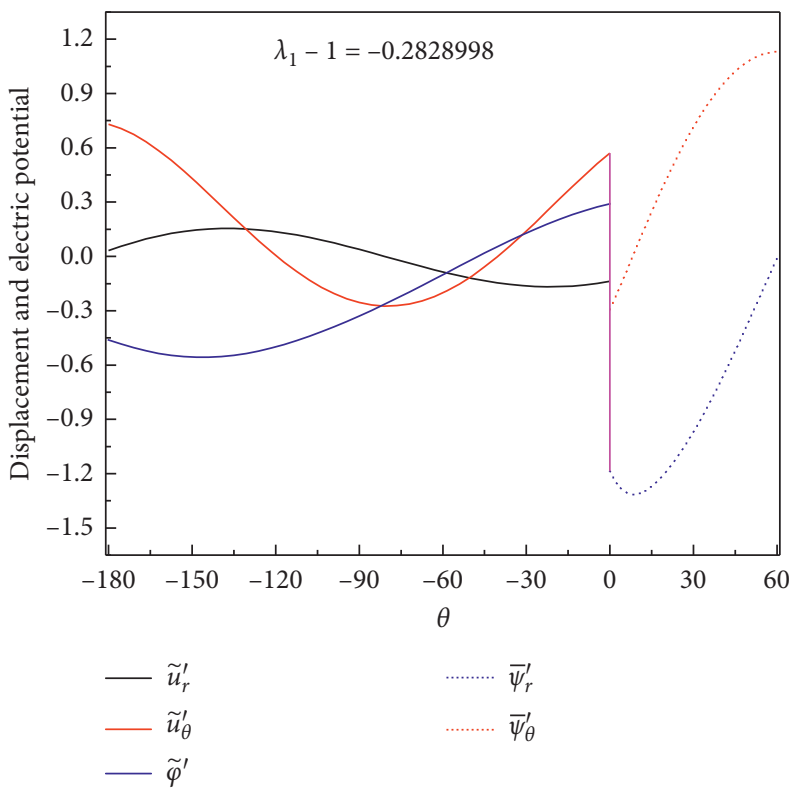

FIgURE 7: The first derivative characteristic angular function of displacement and electric potential.

indicate that the displacement field and potential field in the bonding position of piezoelectric composite material junctions are continuous, but the stress and electric displacement are not equal, which is one of the reasons for the failure and damage of piezoelectric and composite material junctions.

Figure 8 (a) shows the geometry for piezoelectric composite material junctions at $\phi_{1}=90^{\circ}, \phi_{2}=90^{\circ}$, and $\beta=0^{\circ}$ and fiber direction $0^{\circ} \leq \gamma \leq 180^{\circ}$. The singularity orders of electromechanical coupling field at the tip of PZT-4 and Gr/Ep junctions are calculated by the interpolation matrix method at $N=40$. The variation of singularity orders $\lambda_{1}-1$ with the fiber direction $\gamma$ is plotted in Figure 9. It is found that there is only first stress and electric displacement singularity order at the tip of bimaterial junctions at $\phi_{1}=\phi_{2}=90^{\circ}$ and $0^{\circ} \leq \gamma \leq 180^{\circ}$. Besides, the singularity at the tip of junctions is the strongest at $\gamma=135^{\circ}$, in which $\lambda_{1}-1=-0.0964289$.

Figure 8(b) shows the geometry for piezoelectric composite material junctions at $\phi_{1}=90^{\circ}$ and $\phi_{2}=180^{\circ}$ and fiber direction $0^{\circ} \leq \gamma \leq 180^{\circ}$. The variation of singularity orders $\lambda-$ 1 with the fiber direction $\gamma$ is plotted in Figure 10. Figure 10 illustrates that there are second stress and electric 


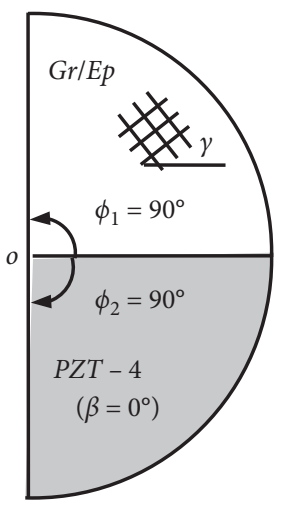

(a)

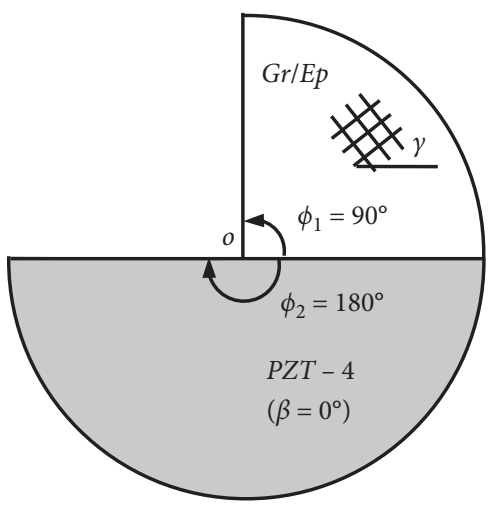

(b)

Figure 8: Geometry for piezoelectric-composite wedge junctions: (a) $\phi_{1}=90^{\circ}, \phi_{2}=90^{\circ}$, and $\beta=0^{\circ}$ and (b) $\phi_{1}=90^{\circ}$ and $\phi_{2}=180^{\circ}$.

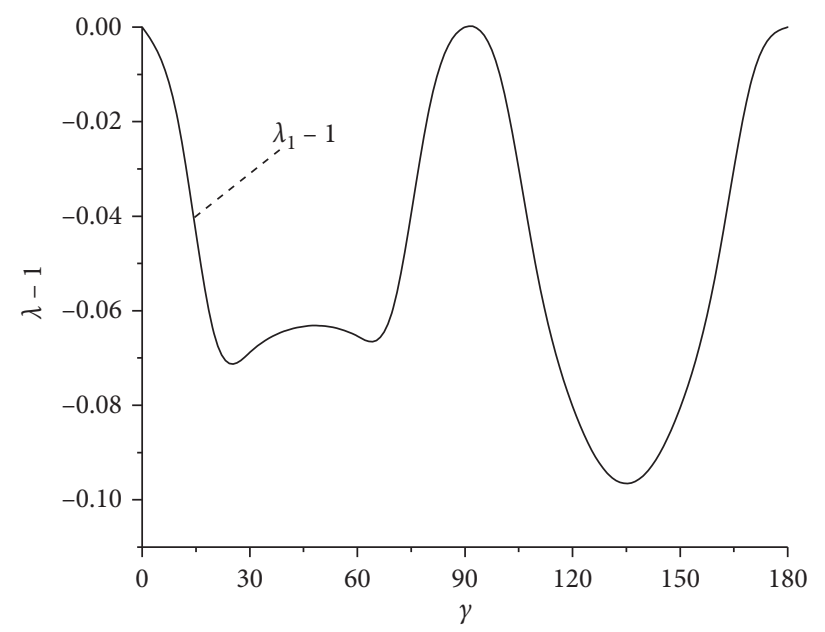

Figure 9: The variations of singularity orders with the fiber direction $\gamma$ of graphite/epoxy at $\phi_{1}=\phi_{2}=90^{\circ}$.

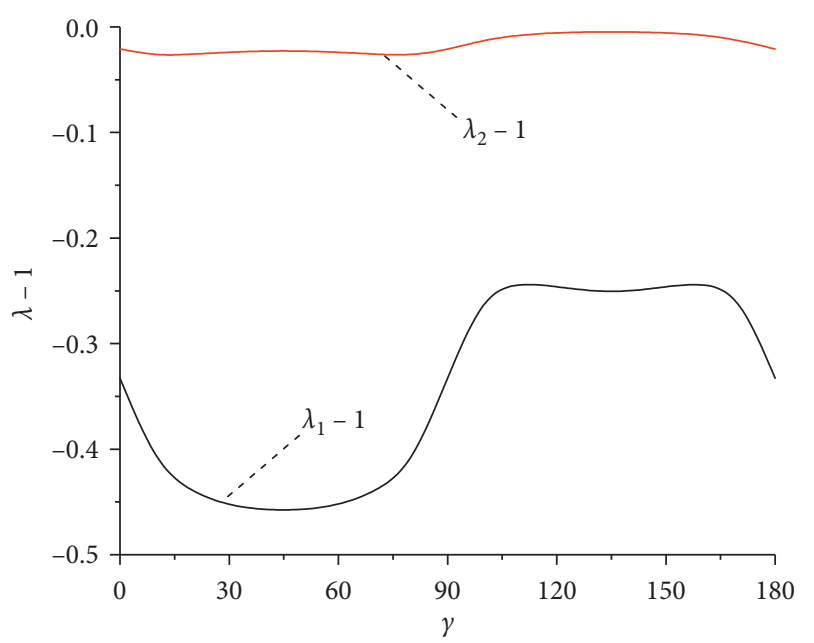

FIGURE 10: The variations of singularity orders with the fiber direction $\gamma$ of graphite/epoxy at $\phi_{1}=90^{\circ}$ and $\phi_{2}=180^{\circ}$.

displacement singularity order at the tip of bimaterial junctions at $\phi_{1}=90^{\circ}$ and $\phi_{2}=180^{\circ}$ and $0^{\circ} \leq \gamma \leq 180^{\circ}$. The fiber direction $\gamma$ has a great influence on the first singularity order $\lambda_{1}-1$, but a little influence on the second singularity order $\lambda_{2}-1$. Besides, the singularity at the tip of junctions is the strongest at $\gamma=45^{\circ}$, where $\lambda_{1}-1=-0.4575995$. 


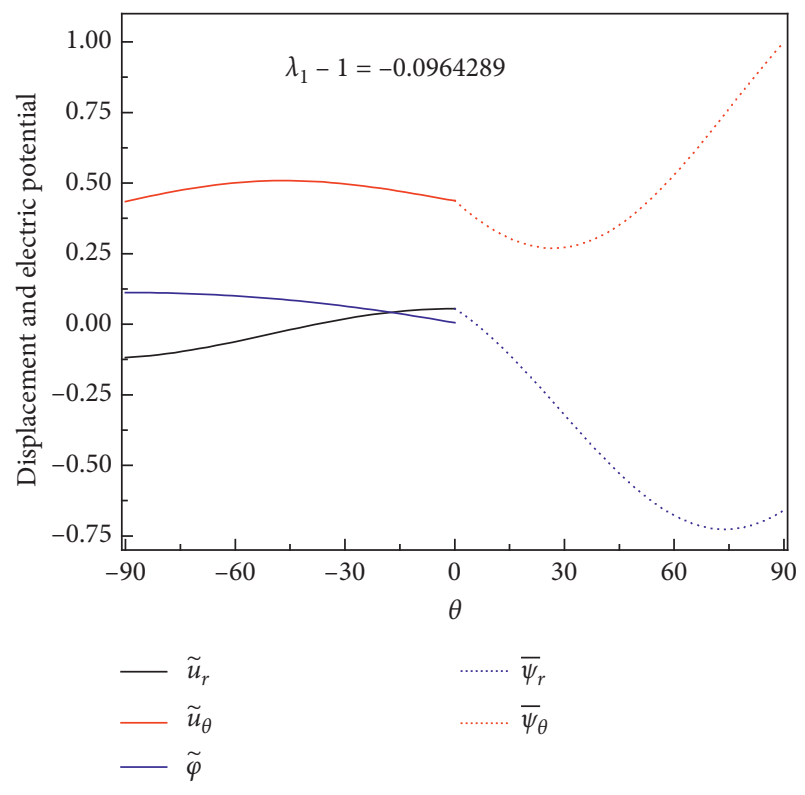

FIGURE 11: The characteristic angular function of displacement and electric potential at $\lambda_{1}-1=-0.0964289$.

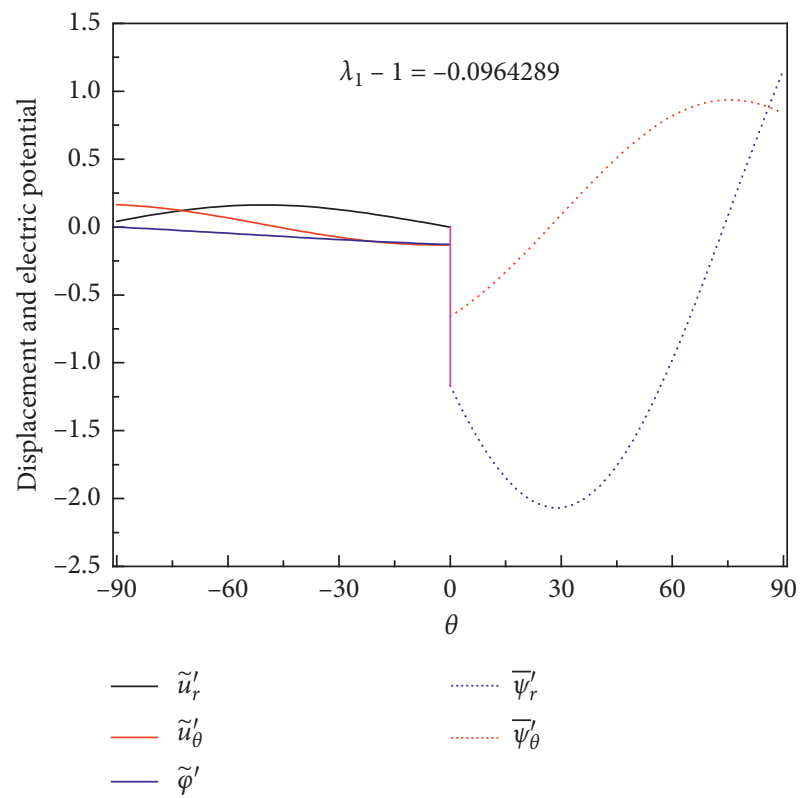

FIGURE 12: The first derivative characteristic angular function of displacement and electric potential at $\lambda_{1}-1=-0.0964289$.

Figures 11 and 12 show the characteristic angular function of displacement and electric potential and their first derivative function at $\phi_{1}=\phi_{2}=90^{\circ}$ and $\gamma=135^{\circ}$, where the first stress singularity order at the tip of PZT -4 and Gr/Ep junctions $\lambda_{1}-1=-0.0964289$. Figures 13 and 14 show the characteristic angular function of displacement and electric potential and their first derivative function at $\phi_{1}=90^{\circ}$, $\phi_{2}=180^{\circ}$, and $\gamma=45^{\circ}$, where the first stress singularity order at the tip of $\mathrm{PZT}-4$ and $\mathrm{Gr} / \mathrm{Ep}$ junctions $\lambda_{1}-1=-0.4575995$.

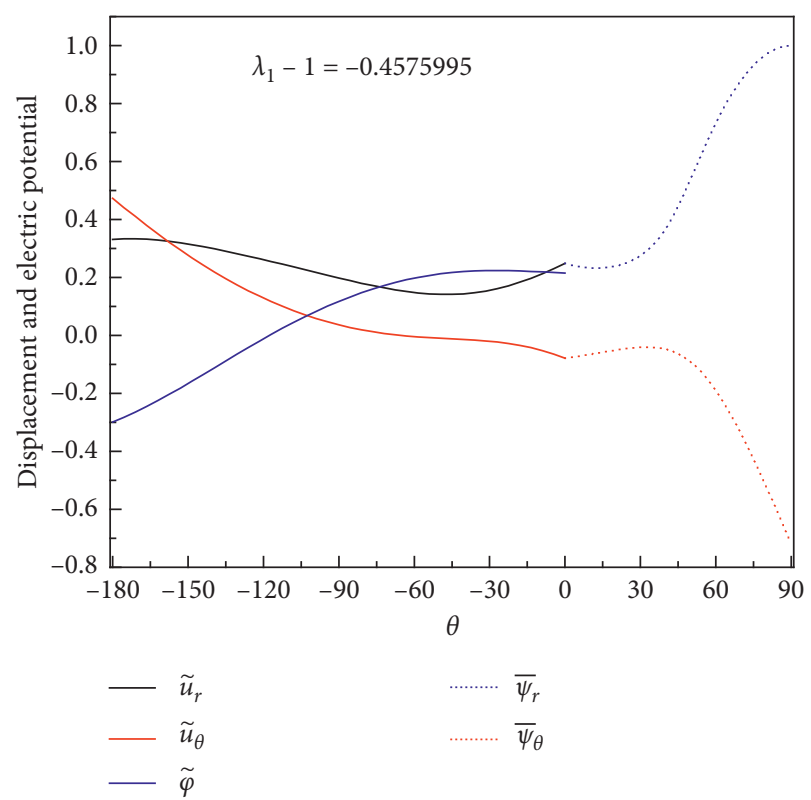

Figure 13: The characteristic angular function of displacement and electric potential at $\lambda_{1}-1=-0.4575995$.

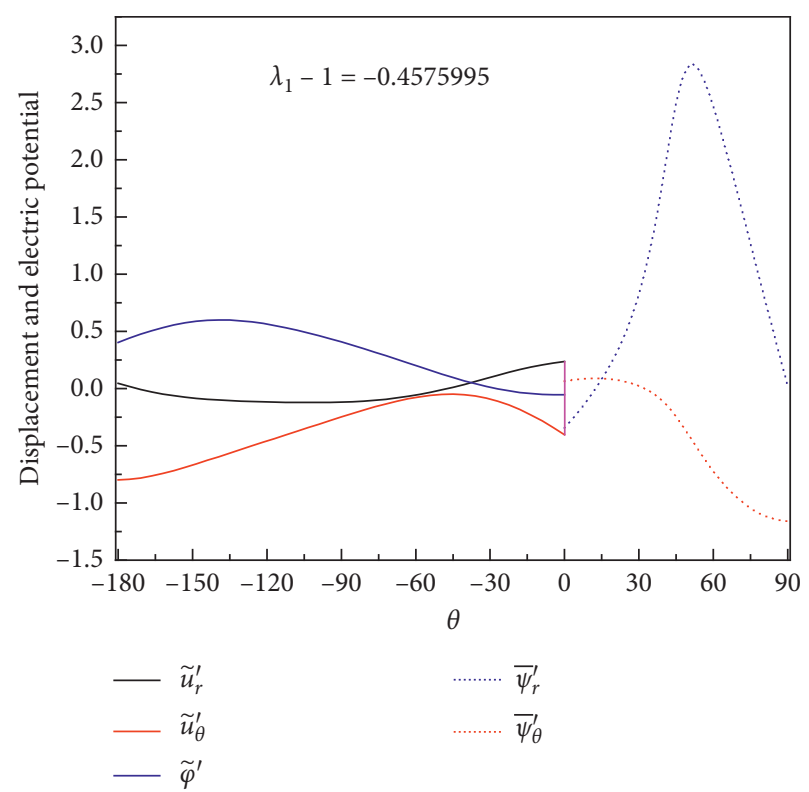

FIgURE 14: The first derivative characteristic angular function of displacement and electric potential at $\lambda_{1}-1=-0.4575995$.

\section{Conclusions}

The numerical method based on the interpolation matrix method was presented to obtain the first couple of singularity orders of electromechanical coupling field at the tip of piezoelectric composite material junctions. The corresponding characteristic angular functions of displacement and electric potential and their derivative functions of each order were obtained at the same time. All these calculated values have the same accuracy, which is a 
significant advantage. Moreover, the desired calculating accuracy can be achieved by subdividing the interval mesh. The main conclusions are summarized as follows:

(1) The singularity orders at the tip of piezoelectric composite material junctions obtained in this paper are in good agreement with the calculated results in the existing literature. The effectiveness and high accuracy of the presented method are proved in calculating the singularity orders of the stress field and electric field at the tip of piezoelectric composite material junctions.

(2) The singularity of the electromechanical coupling field at the tip of piezoelectric composite material junctions is closely related to the bonding angle and fiber direction. The numerical results can be used to guide the optimal configuration for the combination of dissimilar materials.

(3) Based on the results of this paper, the complete stress field and electric displacement field can be obtained by using the finite element or boundary element method for the region around the tip of piezoelectric composite material junctions.

\section{Data Availability}

All data generated or analyzed during this study are included in this article.

\section{Conflicts of Interest}

The authors declare that they have no conflicts of interest.

\section{Acknowledgments}

This research was funded by the Anhui Key University Natural Science Foundation (grant no. KJ2019A0159), Anhui Provincial Natural Science Foundation (grant no. 1808085ME147), and Key Project of Natural Science Foundation of Anhui Polytechnic University (grant no. Xjky110201907).

\section{References}

[1] J.-Q. Xu and Y. Mutoh, "Singularity at the interface edge of bonded transversely isotropic piezoelectric dissimilar materials," JSME International Journal Series A, vol. 44, no. 4, pp. 556-566, 2001.

[2] M. Denda and J. Lua, "Development of the boundary element method for 2D piezoelectricity," Composites Part B: Engineering, vol. 30, no. 7, pp. 699-707, 1999.

[3] M. L. Williams, "The stresses around a fault or crack in dissimilar media," Bulletin of the Seismological Society of America, vol. 49, pp. 199-204, 1959.

[4] T.-Y. Zhang, G. Liu, and Y. Wang, "Failure behavior and failure criterion of conductive cracks (deep notches) in piezoelectric ceramics II: experimental verification," Acta Materialia, vol. 52, no. 7, pp. 2025-2035, 2004.

[5] C.-I. Liu and C.-H. Chue, "On the stress singularity of dissimilar anisotropic wedges and junctions in antiplane shear," Composite Structures, vol. 73, no. 4, pp. 432-442, 2006.

[6] F. Delale and F. Erdogan, "Bonded orthotropic strips with cracks," International Journal of Fracture, vol. 15, pp. 343364, 1979.

[7] D.-H. Chen and K. Harada, "Stress singularities for crack normal to and terminating at bimaterial interface on orthotropic half-plates," International Journal of Fracture, vol. 81, no. 2, pp. 147-162, 1996.

[8] Z. H. Zhou, X. S. Xu, and A. Y. T. Leung, "The mode III stress/electric intensity factors and singularities analysis for edge-cracked circular piezoelectric shafts," International Journal of Solids and Structures, vol. 46, no. 20, pp. 3577-3586, 2009.

[9] Z. Zhou, Z. Yang, W. Xu, X. Yu, C. Xu, and X. Xu, "Evaluation of electroelastic singularity of finite-size V-notched one-dimensional hexagonal quasicrystalline bimaterials with piezoelectric effect," Theoretical and Applied Fracture Mechanics, vol. 100, pp. 139-153, 2019.

[10] C. H. Xu, Z. H. Zhou, X. S. Xu, and A. Y. T. Leung, "Electroelastic singularities and intensity factors for an interface crack in piezoelectric-elastic bimaterials," $A p$ plied Mathematical Modelling, vol. 39, no. 9, pp. 27212739, 2015.

[11] Z. Zhou, X. Xu, A. Y. T. Leung, and Y. Huang, "Stress intensity factors and T-stress for an edge interface crack by symplectic expansion," Engineering Fracture Mechanics, vol. 102, pp. 334-347, 2013.

[12] K. Wippler and M. Kuna, "Crack analyses in three-dimensional piezoelectric structures by the BEM," Computational Materials Science, vol. 39, no. 1, pp. 261-266, 2007.

[13] M. H. Shen, F. M. Chen, and S. N. Chen, "Piezoelectric study on singularities interacting with interfaces in an anisotropic media," International Journal of Solids and Structures, vol. 44, no. 17, pp. 5598-5610, 2007.

[14] X.-L. Xu and R. K. N. D. Rajapakse, "On singularities in composite piezoelectric wedges and junctions," International Journal of Solids and Structures, vol. 37, no. 23, pp. 3253-3275, 2000.

[15] M.-C. Chen, J.-J. Zhu, and K. Y. Sze, "Electroelastic singularities in piezoelectric-elastic wedges and junctions," Engineering Fracture Mechanics, vol. 73, no. 7, pp. 855-868, 2006.

[16] C. Li, H. Man, C. Song, and W. Gao, "Analysis of cracks and notches in piezoelectric composites using scaled boundary finite element method," Composite Structures, vol. 101, pp. 191-203, 2013.

[17] C. Luangarpa and H. Koguchi, "Evaluation of intensities of singularity at three-dimensional piezoelectric bonded joints using a conservative integral," European Journal of Mechanics-A/Solids, vol. 72, pp. 198-208, 2018.

[18] M. S. Islam, M. G. Kader, and M. M. K. Uddin, "Investigation of order of singularity in 3D two-phase transversely isotropic piezoelectric dissimilar joints by eigen analysis method," Procedia Engineering, vol. 90, pp. 58-64, 2014.

[19] M. Hrstka, T. Profant, and M. Kotoul, "Electro-mechanical singularities of piezoelectric bi-material notches and cracks," Engineering Fracture Mechanics, vol. 216, p. 106484, 2019.

[20] Z. Niu, D. Ge, C. Cheng, J. Ye, and N. Recho, "Evaluation of the stress singularities of plane $\mathrm{V}$-notches in bonded dissimilar materials," Applied Mathematical Modelling, vol. 33, no. 3, pp. 1776-1792, 2009.

[21] C. Z. Cheng, S. L. Yao, Z. L. Han, N. Recho, and Z. R. Niu, "Evaluation of the singularity exponents and characteristic angular functions for piezoelectric V-notches under in plane 
and out of plane conditions," Theoretical and Applied Fracture Mechanics, vol. 76, pp. 50-59, 2015.

[22] C. Cheng, X. Cheng, Z. Niu, and N. Recho, "Singularity characteristic analyses for magneto-electro-elastic V-notches," European Journal of Mechanics-A/Solids, vol. 57, pp. 59-70, 2016.

[23] C. Z. Cheng, S. Y. Ge, S. L. Yao, Z. R. Niu, and N. Recho, "Singularity analysis for a V-notch with angularly inhomogeneous elastic properties," International Journal of Solids and Structures, vol. 78-79, pp. 138-148, 2016.

[24] C. Z. Cheng, Z. R. Niu, and N. Recho, "Effect of non-singular stress on the brittle fracture of V-notched structure," International Journal of Fracture, vol. 174, no. 2, pp. 127-138, 2012. 